\title{
Review on the Application of Food Nanotechnology in Food Processing
}

\author{
Mulugeta Wegari Dera ${ }^{1}$, Wondemu Bogale Teseme ${ }^{2}$ \\ ${ }^{1}$ Department of Food Process Engineering, Faculty of Engineering, Gambella University, Gambella, Ethiopia \\ ${ }^{2}$ Department of Food Technology and Process Engineering, Institute of Technology, Haramaya University, Dire Dawa, Ethiopia
}

Email address:

deramulgeta19@gmail.com (M. W. Dera),wondimubogale080@gmail.com (W. B. Teseme)

\section{To cite this article:}

Mulugeta Wegari Dera, Wondemu Bogale Teseme. Review on the Application of Food Nanotechnology in Food Processing. American Journal of Engineering and Technology Management. Vol. 5, No. 2, 2020, pp. 41-47. doi: 10.11648/j.ajetm.20200502.12

Received: February 3, 2020; Accepted: February 24, 2020; Published: May 28, 2020

\begin{abstract}
Nanotechnology is the manufacture, use of materials and structures at the nanometer scale (a nanometre is one millionth of a millimetre). It offers a wide range of opportunities for the development of innovative products and applications in food system. Food quality monitoring using biosensors- intelligent, active and smart food packaging systems; nano emulsions and nano encapsulation of bioactive food compounds are some examples of emerging applications of nanotechnology in the food sector. In food engineering, two major applications related to nanotechnology i.e. food Nano sensing and food nano structured ingredients are being expected. In the former field, better food quality and safety evaluation can be achieved by using nanotechnology. Various types of nano sensors are being developed to meet the different requirements in food inspection (nano sensors for detection of external and internal conditions in food packaging, carbon nanotubes based electrochemical sensors for detection of cations, anions and organic compounds in food, various aptamers for detection of pesticides, antibiotics, heavy metals, microbial cells and toxins). Nanotechnology applications in the food industry can be utilized to detect bacteria in packaging, or produce stronger flavors and color quality, and safety by increasing the barrier properties. However, in the pursuit of delivering patentable technologies, concerns over consumer health and safety in the use of nanoparticles in foods is an ongoing challenge.
\end{abstract}

Keywords: Emulsion, Nano Emulsion, Nano Encapsulation, Nano Sensors, Nanotechnology

\section{Introduction}

The term 'nano' is derived from the Greek word for dwarf [1]. The term "Nanotechnology" was first used in 1974 by the late Norio Taniguchi and concepts were given by Richard Feynman in 1959. A nanometer is a thousandth of a thousandth of a thousandth of a meter $\left(10^{-9} \mathrm{~m}\right)$. One nanometer is about 60,000 times smaller than a human hair in diameter or the size of a virus, a typical sheet of paper is about $100,000 \mathrm{~nm}$ thick, a red blood cell is about 2,000 to $5,000 \mathrm{~nm}$ in size, and the diameter of DNA is in the range of $2.5 \mathrm{~nm}$. Nanotechnology deals with matter that ranges from one half the diameter of DNA up to $1 / 20$ the size of a red blood cell [2]. Nanotechnology is a multidisciplinary technological and scientific field undergoing a rapid development. Research in biology, chemistry, engineering and physics has developed new materials using nanotechnology.
Nanotechnology is one of the emerging food preservation technologies in food industries; it focuses on the characterization, fabrication, and manipulation of biological and non-biological structures smaller than $100 \mathrm{~nm}$. Structures on this scale have been shown to have unique and novel functional properties. Consequently, interest and activities in this research area have greatly increased over the past years. Nano science is defined as the study of phenomena and the manipulation of materials at the atomic, molecular and macromolecular scales, where the properties differ from those at a larger scale [3].

Nanotechnology is defined as the design, production and application of structures, devices, and systems through control of the size and shape of the material at the nanometer $\left(10^{-9}\right.$ of a meter) scale where unique phenomenon enable novel applications [4].

In food and agricultural systems nanotechnologies cover many aspects, such as food safety, packaging materials, 
disease treatment, delivery systems, bioavailability, new tools for molecular and cellular biology, and new materials for pathogen detection [5]. It is likely that it is only a matter of time until we see the products of nanotechnology "on our planet. In food and dairy industries, the applications of nanotechnology include Nano particulate delivery Systems (nano dispersions and nano capsules), Packaging (nano laminates, nano composites bottles, bins with silver nanoparticles), Food Safety and Biosecurity (nano sensors) etc [6]. The nanotechnology will play an vital role in the food and dairy processing in near future and would involve two forms of nano food applications viz, food additives (nano inside) and food packaging (nano outside). The nano scale food additives may be used to influence texture, flavour, nutritious improvement, provide functionally and even detect pathogens and food packaging involves extend food shelf life, edible, nano wrapper which will envelope foods, preventing gas and moisture exchange, 'smart' packaging (containing nano-sensors and anti-microbial activators) for detecting food spoilage and releasing nano-anti-microbes to extend food shelf life [7].

Objective

The aim of this review was;

a) To review the emerging food preservation technology and food nanotechnology, and

b) To review the Application and function of different nanotechnologies in food industries

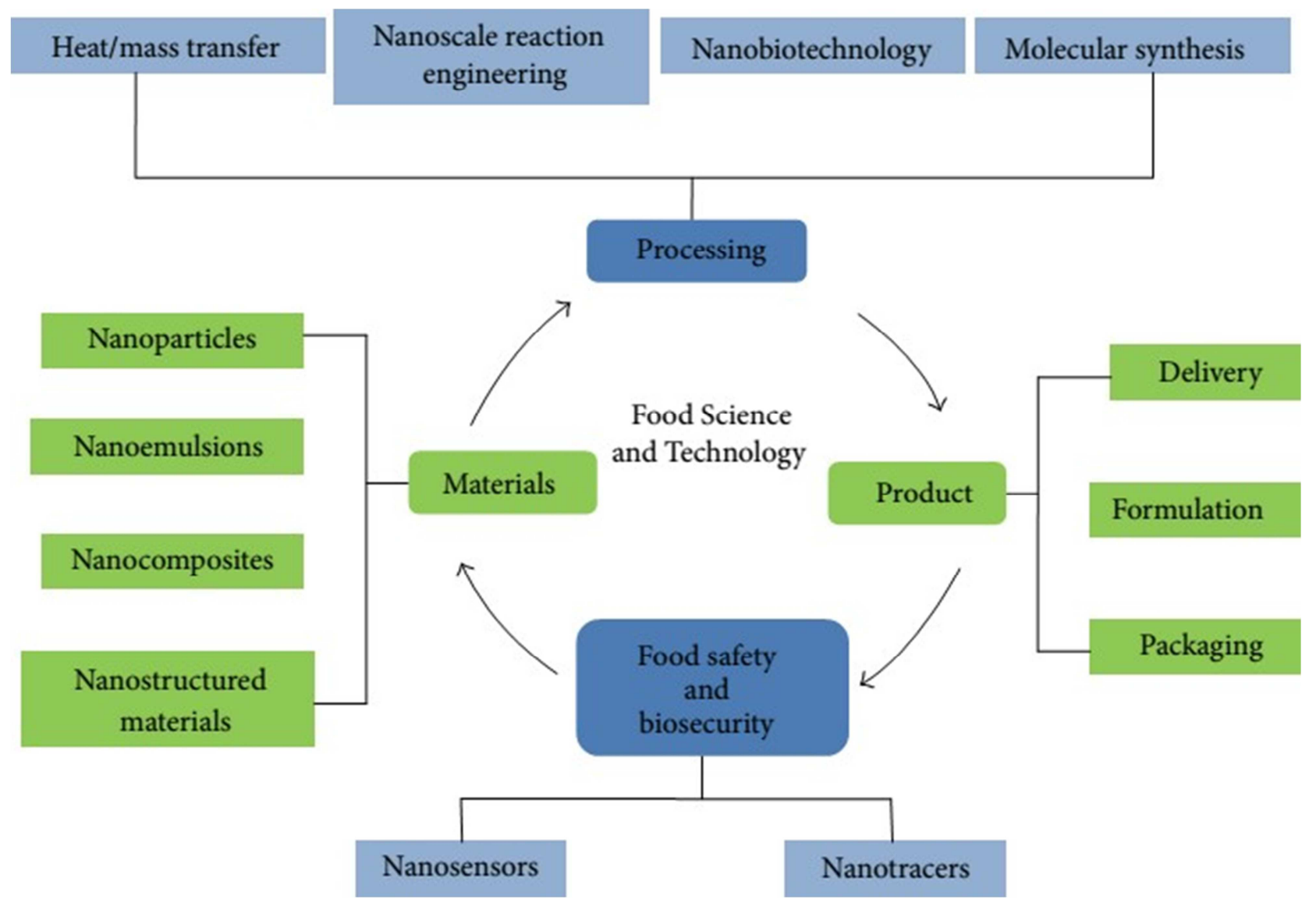

Figure 1. Different steps of food management and the contribution of nanotechnology.

\section{Literature Review}

\subsection{Nanotechnology in Food Processing}

Food processing can be defined as a practice of preserving food with the help of methods and techniques in order to transform food to a consumable state. These techniques are designed as such that the flavor and quality of the food are kept intact but they are also protected from infestation of microorganisms that leads to food spoilage. Irradiation, ohmic heating, and high hydrostatic pressure are few of the conventional methods of food processing. Food processing methods that involve the nano materials include incorporation of nutraceuticals, gelation and viscosifying agents, nutrient delivery, mineral and vitamin fortification, and nano encapsulation of flavors. Processing of food is mainly carried out in order to keep the food intact and also to increase its shelf life [8].

Enzymes can be applied in some food processing methods for changing food components to enhance flavor, nutritional value, health benefits, etc. The use of nano materials provides superior enzyme support systems (improving activity, shelf life, and cost-effective) due to their help in dispersion through food matrices and their large surface-to-volume ratios compared to traditional macro scale support materials. For example, nano-silicon dioxide particles effectively hydrolyzed olive oil with modified stability, adaptability, and reusability [9].

\section{Nanobiotechnology Molecular synthesis}




\subsection{Nanotechnology in Food Packaging and Food Preservation}

Food packaging methods are used to make sure that the quality of the food is kept intact however; they are packaged in a way so that it is safe for consumption. Packaging mainly aims at providing physical protection in order to prevent the food from external shocks and vibration, microbial infestation, and temperature in providing barrier protection by scavenging oxygen and other spoilage causing gases. The packaging materials are preferably made of biodegradable materials in order to reduce environmental pollution. This idea has been turned into reality due to the introduction of nanotechnology in food packaging industry. High barrier plastics, introducing antimicrobials, and detection measures for contaminants are few of the methods that require being paid attention to while food is being packaged [9].

Whereas treating and handling of food in order to slow down the spoilage, resulting in the prevention of loss of food quality, edibility, or nutritive value by the microorganisms, are termed as food preservation. Drying, canning, and freezing are few of the conventional methods that have found their use as food preservation techniques. Food is managed in several different steps that involve processing, packaging and preservation means at the same time. Each of these steps is assisted by nanotechnology with the help of several nano materials [9].

\subsubsection{Edible Thin Film Packaging}

The use of edible thin film or packages can delay food deterioration by increasing shelf life and enhancing quality. Carrageenan, chitosan, gelatin, poly lactic acid, polyglycolic acid, alginate, blends of starch and sodium caseinate are some material used in the production of bio plastic with application in edible thin film packaging. The edible thin films can be used in extended applications, for example, to protect fruit, vegetables, meats, chocolate, candies, baked goods and French fries, because these films act as active packaging increasing barrier protection by preventing gases such as oxygen and ethylene from damaging the food content and preserving the appearance of the product [10].

The edible thin film can be prepared from edible nano laminates that might protect the food from moisture, lipids, gases, off-flavors and odors. These materials can be prepared with polysaccharides, proteins or lipids. The polysaccharide and protein films are good barriers against oxygen and carbon dioxide, but these films are not efficient to protect against moisture. Lipid films are good for this task, but they offer limited resistance to gases and have poor mechanical strength. It is possible to add charged lipid or colloidal particles to edible films as nano laminates that could include various functional agents such as antimicrobials, antibrowning agents, antioxidants, enzymes, flavours and colors. A nano layered film was produced using two polysaccharides with opposite charges, chitosan and sodium alginate deposited onto amino lysed/charged [10].

\subsubsection{Nano Encapsulation}

Nano encapsulation is carried out with the help of nano capsules. They provide several benefits such as ease of handling, enhanced stability, protection against oxidation, retention of volatile ingredients, taste making, moisture triggered controlled release, $\mathrm{pH}$ triggered controlled release, consecutive delivery of multiple active ingredients, change in flavor character, long lasting organoleptic perception, and enhanced bioavailability and efficacy. They can be defined as Nano vesicular systems that exhibit a typical core-shell structure in which the drug is confined to a reservoir or within a cavity surrounded by a polymer membrane or coating. The cavity can contain the active substance in liquid or solid form or as a molecular dispersion. Nano capsules are involved in the delivery of the desired component and entrapment of the odor and unwanted components in the food and thereby resulting in the preservation of the food [9]. In the biological system, nano capsules carry the food supplements via the gastrointestinal tract and this leads to increased bioavailability of the substance. There are six basic ways of preparation of nano capsules, namely:

a) Nano precipitation,

b) Emulsion-diffusion,

c) Double emulsification,

d) Polymer coating, and

e) Layer by-layer

The basic difference between a conventional emulsion and nano emulsion is that a nano emulsion does not change the appearance of the food item when added to it. These nano capsules find their use in the delivery of pesticides, fertilizers and vaccines to the plants. They are also used to deliver lipophilic health supplements such as vitamin and minerals in the food, fatty acids, and growth hormones, increasing the nutrient content of the food. The basic benefit of encapsulation is to protect the hidden component to deliver it precisely at the target even in unfavorable conditions.

Liposome is an example of a nano-based carrier used for nano encapsulation. Nano liposomes help in controlled and specific delivery of the several components within the system. They are known to deliver nutraceuticals, nutrients, enzymes, vitamins, antimicrobials, and additives [11]. Zein fibers loaded with gallic acid using electro spinning are a new method of encapsulation technique where research is being carried out [12]. Zein fiber protects the lipids from being degraded within the system before it reaches the target delivery. This new, effective method can actually be utilized thoroughly by the food packaging industry. Lipid based encapsulation systems are much more efficient in comparison to other encapsulation systems because of the better solubility and specificity of the components encapsulated within it.

\subsubsection{Nano Emulsion}

Nano emulsions are used to produce food products for salad dressing, flavored oils, sweeteners, personalized beverages, and other processed foods. They help in releasing different flavors with the help of several stimulations in the 
form of heat, $\mathrm{pH}$, ultrasonic waves, and so forth. They retain the flavors efficiently and prevent them from oxidation and enzymatic reactions. Nano emulsions created mainly by two approaches;

1. High energy approach involves the steps of high pressure homogenization, ultra sound method, high-speed liquid coaxial jets and high speed devices method, and

2. Similarly, low energy approach Involves membrane emulsification, spontaneous emulsification, solvent displacement, emulsion inversion point, and phase inversion point.

The nano emulsions created by dispersing liquid phase in continuous aqueous phase. The components that used for the creation of nano emulsion is lipophilic where the lipophilic component mixed thoroughly with the oil phase. The placement of the lipophilic component within the nano emulsion depends on several factors such as molecular and physicochemical properties. The physicochemical property includes hydrophobicity, surface activity, oil-water partition coefficient, solubility, and melting point. Several lipophilic components are encapsulated with the help of nano emulsion formation [9].

For example, $\beta$-carotene, citral, capsaicin, flaxseed oil, tributyrin, coenzyme $\mathrm{Q}$ and several oil soluble vitamins. They are highly stable to gravitational separation and droplet aggregation and nano emulsion is thermally stable in comparison to the conventional emulsions. Nano emulsions are preferred these days rather than the conventional emulsions because the smaller the droplet is, larger the surface area is and the more readily they will be digested by the digestive enzymes and ultimately be absorbed easily [9].

\subsubsection{Nano Sensors}

Nano sensors help in detecting any sort of change in the color of the food and it helps in the detection of any gases being produced due to spoilage. The sensors are usually sensitive towards gases such as hydrogen, hydrogen sulphide, nitrogen oxides, sulphurdioxide and ammonia [9].

They are a device comprising an electronic data processing part and sensing part that is able to detect any change in light, heat, humidity, gas, and chemicals into electrical signals. The high sensitivity and selectivity of the nano sensors make them more efficient than the conventional sensors. These gas sensors are made up of metals such as Palladium, platinum, and gold [9].

Gold-based nanoparticles are also used at times to detect toxins such as aflatoxin B1 in milk. At times, they are even made up of single walled carbon nanotubes and DNA, which increases the sensitivity of the sensors. Liposome nano vesicles have been applied for detection of peanut allergenic proteins in chocolate and pathogens. Using universal protein G-liposomal nano vesicles and an immounomagnetic bead sandwich assay can simultaneously detect E. coli O157:H7, Salmonella spp. and Listeria mono cytogenes [8]. In agriculture, nano sensors help in monitoring the condition of the soil required for the growth of the crop. They also help in detecting the presence of pesticides on the surface of fruits and vegetables. Not only pesticides, but there are also nano sensors that have been developed to detect carcinogens too in food materials.

The commonly used sensors that are used in the food packaging industries are time-temperature integrator and gas detector. Several different types of nano sensors are used for example, array biosensors, nanoparticle in solution, nanoparticle based sensors, electronic noses, nano-test strips, nano cantilevers. Electronic noses are a type of sensor that uses several chemical sensors that is attached to a data processing system. Since the sensor behaves like a human nose, the sensor is known as electronic nose. Along with the electronic nose, there are reports of electronic tongue sensors that work on a similar principle as that of an electronic nose. It changes color on coming in contact with any sign of spoilage in the food material thus declaring that the food is not fit for consumption [9].

\section{Function and Application of Food Nano Technology}

Table 1. Application matrix of nanotechnology in main areas of food science and technology.

\begin{tabular}{|c|c|c|}
\hline Area of application & Purpose and fact & Approaches \\
\hline $\begin{array}{l}\text { Design of } \\
\text { nanomaterial }\end{array}$ & $\begin{array}{l}\text { Nanoparticles, Nano emulsions, } \\
\text { Nano composites, Nano bio- } \\
\text { composites } \\
\text { (nano bio polymeric starch) Nano } \\
\text { laminates }\end{array}$ & $\begin{array}{l}\text { Novel defined material, with self-assembling, self-healing, and manipulating } \\
\text { Properties }\end{array}$ \\
\hline $\begin{array}{l}\text { Nano sensors and } \\
\text { Nano biosensors }\end{array}$ & Quality control and food safety & $\begin{array}{l}\text { Detection of very small amounts of chemical contaminants monitoring and tagging of food } \\
\text { items } \\
\text { Electronic nose and tongue for sensor evaluation } \\
\text { Food born pathogen identification by measurement of nucleic acid, protein or any other } \\
\text { indicator metabolite of microorganism }\end{array}$ \\
\hline Processing & $\begin{array}{l}\text { Nano filtration } \\
\text { Nano scale enzymatic reactor } \\
\text { Heat and mass transfer } \\
\text { Nanofabrication } \\
\text { Nano capsules for modification of } \\
\text { absorption }\end{array}$ & $\begin{array}{l}\text { Selective passage of materials on the basis of shape and size Improved understanding of } \\
\text { process } \\
\text { Enhanced heat resistance of packages } \\
\text { Nano ceramic pan to reduce time of roasting and amount of consumed oil, reduction of } \\
\text { trans fatty acids due to usage of plant oil instead of hydrogenated oil and finally resulted in } \\
\text { safe nano food development of nano capsules that can be incorporated into food to deliver } \\
\text { nutrients to enable increased absorption of nut }\end{array}$ \\
\hline New products & $\begin{array}{l}\text { Packaging } \\
\text { Delivery }\end{array}$ & $\begin{array}{l}\text { Nano composites application as barriers, coating, release device, and novel packaging } \\
\text { modifying the permeation behavior of foils, increasing barrier properties (mechanical, }\end{array}$ \\
\hline
\end{tabular}




\begin{tabular}{lll}
\hline Area of application & Purpose and fact & Approaches \\
\hline & Formulation & thermal, chemical, and microbial), improving mechanical and heat-resistance properties, \\
& Evaluation & developing active antimicrobial surfaces, sensing as well as signaling microbiological and \\
DNA recombinant technology & biochemical changes, developing dirt repellent coatings for packages \\
& Nano mycells for targeted delivery of nutrients (nutrition nano therapy) \\
& Nano capsulation for controlled release of nutrients, proteins, antioxidants, and flavors \\
& Production of nano scale enzymatic reactor for development of new product. \\
& Fortification of food by omega3 fatty acid, haem, licopene, beta-caraton, phitosterols, \\
& DHA/EPA \\
& Enzyme and protein evaluation as nano biological system to development of new products \\
& Recombinant enzyme production in nano porous media with special numerous application. \\
\hline
\end{tabular}

\subsection{Protection Against Biological Deterioration}

\subsubsection{Antimicrobials}

Microbial contamination has been leading to pathogenic infections and poor nutrition associated with weaning foods. Thus, dealing with bacterial deterioration is one of the most critical subjects in the production, processing, transport, and storing of food. Novel nano antimicrobials have shown promising effects on safeguarding food deterioration, thereby extending the shelf life of food. A number of metal and metal oxide nano materials have long been suggested to be effective's antimicrobials. Their intrinsic physicochemical properties allow excessive formation of reactive oxygen species (ROS), leading to oxidative stress and subsequent cell damage [13].

\subsubsection{Increasing Bioavailability}

Nano delivery systems can increase the bioavailability of bio actives in various ways.

\section{$\mathrm{BA}=\mathrm{B} * \mathrm{~A} * \mathrm{~T} *$}

Where $\mathrm{BA}$ is the oral bioavailability of bioactive compounds, $\mathrm{B}^{*}$ is bio accessibility, $\mathrm{A}^{*}$ is absorption, and $\mathrm{T}^{*}$ is molecular transformation. Thus, in order to maximize the bioavailability of bio actives, one can improve the bio accessibility and absorption, and alter the molecular structure that might have occurred during digestion. By altering particle size, solubility can be improved through an increase of the surface area-to-volume ratio, leading to an increase in bio accessibility [13].

\subsection{Protection Against Chemical Ingredients}

\subsubsection{Antioxidants}

Although some metal/metal oxide nano materials are known to cause oxidative stress via formation of ROS, less reactive nano materials are developed to act as antioxidant carriers. Polymeric nanoparticles are suggested to be suitable for the encapsulation of bioactive compounds (e.g., flavonoids and vitamins) and to release them in acidic environments (i.e., stomach). Similarly, $\mathrm{SiO}_{2}$ egallic acid nanoparticles as novel nano antioxidants are developed and tested based on its scavenging capacity of 2, 2-diphenyl-1picrylhydrazyl radicals [13].

\subsubsection{Flavors}

As one of most important parts of the food system, flavors deliver sensory perception of taste and smell to enhance the overall eating experience. Nano encapsulation techniques have widely been used to improve flavor release and flavor retention, and to deliver culinary balance. It has also been shown that $\mathrm{SiO}_{2}$ nanomaterials can act as carriers of fragrances or flavors in food and nonfood products [14].

\subsection{Enhancement of Physical Properties}

\subsubsection{Color Additives}

Certain nanomaterial products have currently been approved for use as food color additives, which have a vital role in the psychological appeal of consumer products. The U.S. FDA approved $\mathrm{TiO}_{2}$ as a food color additive with the stipulation that the additive should not to exceed $1 \% \mathrm{w} / \mathrm{w}$ and now are exempted from certification. Color additive mixtures for food use made with $\mathrm{TiO}_{2}$ may also contain $\mathrm{SiO}_{2}$ and/or $\mathrm{Al}_{2} \mathrm{O}_{3}$, as dispersing aids do not more than $2 \%$ total. However, the use of carbon black as a food color additive is no longer authorized [13].

\subsubsection{Anticaking Agents}

$\mathrm{SiO}_{2}$ used mainly to thicken pastes, as an anticaking agent to maintain flow properties in powdered products, and as a carrier of fragrances or flavors in food and nonfood products. It has widely been applied in food products and registered within the EU as a food additive (E551). Recent research showed that in powdered food materials containing E551, at least a part of the $\mathrm{SiO}_{2}$ is in the nano size range [13].

\subsection{Safety Concern in Food Nanotechnology}

As the investigation into the application of nanotechnology in the food sector increases, the potential of nanotechnology in food science/industry also expands, and consequently so does the human exposure to these substances [15]. It is inevitable that human exposure to nano materials will increase in various ways, either intended or unintended. However, a few studies have focused on the potential toxicity of the presence of nano materials in foods, by analyzing food samples used in food additives/ingredients and food packaging. They know little about the bioavailability, bio distribution, routes of nano materials, and the ultimate toxicity upon exposure. Most noticeably, nano materials, serving as food additives, come in direct contact with human organs. It may result in higher levels of exposure depending on their concentration in food and the amount of that food consumed. Increasing uses of nanomaterial substances in 
foods as flavor or color additives have attracted significant attention of public and government sectors [13].

A study on $\mathrm{TiO}_{2}$ in sugar-coated chewing gum found that over $93 \%$ of $\mathrm{TiO}_{2}$ in gum is of nano size [16]. It is unexpectedly easy for $\mathrm{TiO}_{2}$ to be released, be swallowed by a person chewing the gum, and be accumulated in the body gradually [16]. Similarly, the gut epithelium most likely exposed to $\mathrm{SiO}_{2}$ nanoparticles upon consumption of foods containing E551 [17, 18]. However, none of them provided toxicological studies with respect to nanomaterial exposure. In other cases, exposure may occur unintentionally via leaching from nano packaging. Nano clay from food contact materials was found to migrate in to food simulants.

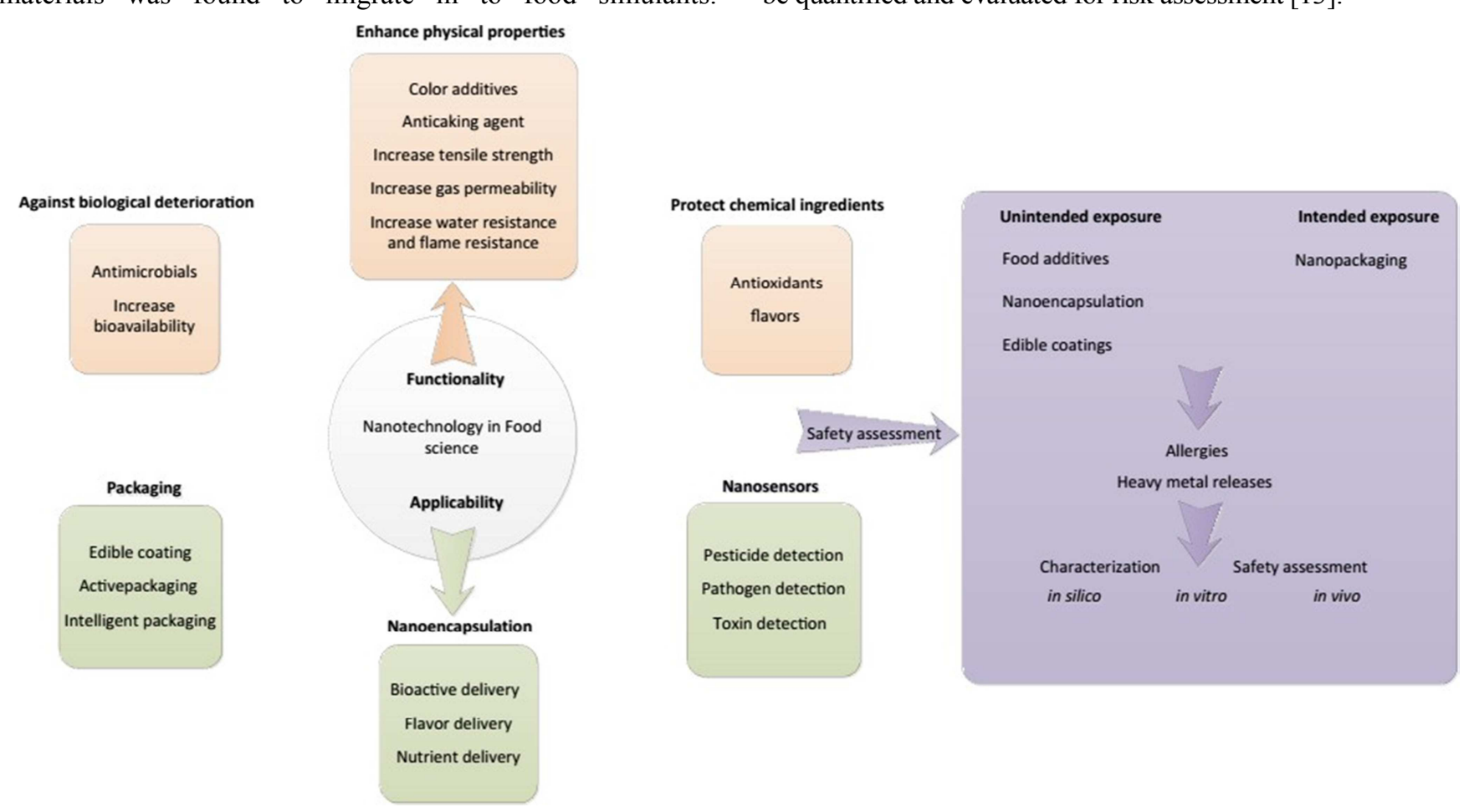

Figure 2. Development of nanotechnology in food industry and its functionality, applicability, and safety assessments.

\section{Conclusion}

Nanotechnology has brought forth a revolutionary effect on the food processing and preservation industry, it is one of the emerging technologies in food industry. There are definite advantages of the technology but the drawbacks are equally prominent. Several food industry giants are paying in millions to develop nano systems that will help preserve the food better. Care should be taken while designing newer nano systems so that they are both environment friendly and they do not have any toxic effect on the food. Thorough testing needs to be carried out in health claims of the products that are being launched. Rather than having a chemical approach towards designing the nano systems, research should be carried out in trying to discover natural nano-systems for the delivery of drug or health supplements through food.

Nanoparticles, being ultramicroscopic in size, are easily taken up by the cells inside the human body and can have toxic effects. The toxicity is in an enhanced fashion due to
Furthermore, it was found that migration of nano clay from the multilayer film into food simulants increased with increasing contact time and temperature [13]. It should be noted that the ultimate fate and toxicity of nano materials in foods and food packaging depend on physiochemical characteristics and dose.

Safe application of nanotechnology to the food industry requires thorough characterization and assessment in silico, in vitro, and in vivo. Altogether, taking into consideration physical forces, osmotic concentration, $\mathrm{pH}$, chemical factors, biological molecules, and commensal microbes, their absorption, distribution, metabolism, excretion, and ultimate toxicity could be quantified and evaluated for risk assessment [13]. 


\section{Acknowledgements}

First, it is our pleasure to express our heart-felt appreciation and special gratitude to our advisor D. Eng Solomon Abera for his unreserved support and supervision during the preparation of this review paper. We treasure his advises which have contributed a great deal to the success of this work.

\section{References}

[1] Momin, J. K. and B. Joshi, Nanotechnology in foods, in Nanotechnologies in Food and Agriculture. 2015, Springer. p. 3-24.

[2] Dingman, J., Guest commentary: nanotechnology: its impact on food safety. Journal of Environmental Health, 2008. 70 (6): p. $47-50$.

[3] Morillon, V., et al., Factors affecting the moisture permeability of lipid-based edible films: a review. Critical reviews in food science and nutrition, 2002. 42 (1): p. 67-89.

[4] Chau, C.-F., S.-H. Wu, and G.-C. Yen, The development of regulations for food nanotechnology. Trends in Food Science \& Technology, 2007.18 (5): p. 269-280.

[5] Maynard, A. D., et al., Safe handling of nanotechnology. Nature, 2006. 444 (7117): p. 267-269.

[6] Chen, H., J. Weiss, and F. Shahidi, Nanotechnology in nutraceuticals and functional foods. Food technology (Chicago), 2006. 60 (3): p. 30-36.

[7] Richardson, N. S. M. a. E. P., Nanotechnology in nutritional sciences. Minerv Biotechnol. 20: 17-126. 2008.

[8] Rashidi, L. and K. Khosravi-Darani, The applications of nanotechnology in food industry. Critical reviews in food science and nutrition, 2011. 51 (8): p. 723-730.
[9] Pradhan, N., et al., Facets of nanotechnology as seen in food processing, packaging, and preservation industry. BioMed research international, 2015. 2015.

[10] Durán, N. and P. D. Marcato, Nanobiotechnology perspectives. Role of nanotechnology in the food industry: a review. International Journal of Food Science \& Technology, 2013. 48 (6): p. 1127-1134.

[11] Godwin, H. A., et al., The University of California Center for the environmental implications of nanotechnology. 2009, ACS Publications.

[12] Thomas, K. and P. Sayre, Research strategies for safety evaluation of nanomaterials, Part I: evaluating the human health implications of exposure to nanoscale materials. Toxicological sciences, 2005. 87 (2): p. 316-321.

[13] He, X. and H.-M. Hwang, Nanotechnology in food science: Functionality, applicability, and safety assessment. journal of food and drug analysis, 2016. 24 (4): p. 671-681.

[14] Dekkers, S., et al., Presence and risks of nanosilica in food products. Nanotoxicology, 2011.5 (3): p. 393-405.

[15] Magnuson, B. A., T. S. Jonaitis, and J. W. Card, A brief review of the occurrence, use, and safety of food-related nanomaterials. Journal of food science, 2011. 76 (6): p. R126R133.

[16] Chen, X. X., et al., Characterization and preliminary toxicity assay of nano-titanium dioxide additive in sugar-coated chewing gum. Small, 2013.9 (9-10): p. 1765-1774.

[17] Peters, R. J., et al., Characterization of titanium dioxide nanoparticles in food products: analytical methods to define nanoparticles. Journal of agricultural and food chemistry, 2014. 62 (27): p. 6285-6293.

[18] Athinarayanan, J., et al., Presence of nanosilica (E551) in commercial food products: TNF-mediated oxidative stress and altered cell cycle progression in human lung fibroblast cells. Cell biology and toxicology, 2014. 30 (2): p. 89-100. 